\title{
Reducing Biases in Cross-Cultural Top Management Team Decision-Making Processes
}

\author{
David Strutton ${ }^{1} \&$ William Carter ${ }^{2}$ \\ ${ }^{1}$ Marketing \& Logistics, University of North Texas, Denton, USA \\ ${ }^{2}$ Department of Management, University of North Texas, USA \\ Correspondence: David Strutton, Professor of Marketing \& Logistics, \& Director, New Product Development \\ Scholars Program, University of North Texas, Denton, TX 76203, USA. Tel: 940-565-3123. E-mail: \\ David.Strutton@unt.edu
}

Received: February 19, 2013

Accepted: April 2, 2013

Online Published: May 12, 2013

doi:10.5430/ijba.v4n3p1

URL: http://dx.doi.org/10.5430/ijba.v4n3p1

\begin{abstract}
Cross-cultural top-management-teams (cc-TMTs) are typically comprised of rational individuals. Yet cc-TMTs featuring uniformly wise and experienced executives often make irrational decisions (i.e., choices incompatible with reason or logic). Even most executives acknowledge this is true. Behavioral economics suggests a primary reason why is that iterative combinations of cultural and cognitive biases invariably arise and interact in cc-TMT settings. As they arise, these biases often undermine the rationality of decision-making processes used inside teams. The impact of such biases surely intensifies if they remain unacknowledged - and thus unmanaged. From an example of Sino and Anglo cc-TMT interactions, six guidelines intended to reduce bias are developed. These approaches, and the analysis preceding them, explain the nature and scope of these cultural and cognitive biases, how and why they arise, and what actions executives might initiate to negate these biases' potentially irrational influence on decision-making processes by consolidating divergent biases to secure mutual gain within cc-TMT settings. Cultural and cognitive biases hardly explain everything, but they explain enough of what happens inside cc-TMTs that ignoring their influence is foolish. This article offers a logical footing from which to begin this important task.
\end{abstract}

Keywords: cultural and cognitive biases, top management teams, behavioral economics, irrational/rational decision-making processes, Sino/Anglo cultural spheres

\section{Introduction}

Five behavioral aspects generally characterize individuals whose reasoning and decision-making facilities are deemed wise by others. Wise-reasoning, psychologists agree, is associated with individuals':

- willingness to seek opportunities to resolve conflict;

- willingness to search for compromise;

- recognition that their personal knowledge is limited;

- acceptance that more than one perspective regarding any problem can exist; and

- understanding that circumstances often worsen before improving, if they improve at all.

The right skills, knowledge and accomplishments surely move managers ahead in their careers. Advancement into top management teams (TMTs) is also spurred by managers' reputations for making rational decisions. And demonstrated tendencies for applying personal experience and the expertise of others when making crucial decisions has likely never restrained anyone's rise. Most TMT participants unquestionably indeed are wise and rational decision makers. But as often happens there is a rub. Here, one associated with four related realities.

The first reality is that even teams comprised of wise and highly experienced executives often make irrational decisions (i.e., choices incompatible with reason or logic). Executives agree; McKinsey reports $72 \%$ admitted their organizations' top decision-making processes were as likely to be flawed as high quality (Lovallo \& Sibony, 2010). Why would the presumably collective wisdom and rationality of executive teams desert them during crucial decision-making episodes? One possible answer, a second reality, suggests executives too often fail to account for cognitive biases that arise during TMT interactions. When intra-team biases go unchecked, their subtle or resounding 
consequences may contribute to TMT participants' systematic tendencies to diverge from their normally rational decision-making processes. They might, for example, fail to seek compromise or resolve conflict; reject others' perspectives outright; or ignore possibilities that conditions can worsen.

A third reality builds on the second. In addition to cognitive biases that often undermine the collective rationality of TMTs, cultural biases exist and may influence how TMT members interpret relevant environments and evaluate decision-making processes. The material consequences associated with cultural biases, and the opportunity to manage them, suggests this reality should be confronted directly. In today's global economy, TMTs increasingly feature extreme levels of cross-cultural complexity (i.e., Chinese-Ghanaian or French-Iranian teams). Hereafter, such cross-cultural top management teams are labeled cc-TMTs.

Like facts, realities are stubborn things; difficult to argue against, overturn or ignore. And no matter what amount of wisdom, experience or due diligence goes into selecting TMT participants, a fourth reality endures: unlike truth, rational decision-making processes do not always "will out" in cc-TMT contexts. Appropriate analyses conducted by rational TMT decision-makers possessing good judgment and sufficient experience will not invariably yield superior decision outcomes.

These four realities coalesce to ground this study. Collectively, they drive the discussion that follows as two questions are addressed:

- Given that executives are usually rational individuals, why does the cohort often engage in decision-making processes that yield irrational outcomes in cc-TMT settings?

- Relatedly, what can be done to mediate cc-TMT irrationality and exploit biases for team advantage?

Each question can be partially answered by considering the presence and influence of culturally-instilled cognitive biases. Culturally-mediated personal, procedural and decision-making biases exist as part of humanity's cognitive "deep structures." Deep structures are psychological schema constructed and reinforced through humans' experiences in their primary cultures and environments. Paradigms, heuristics and stereotypes emerge from these worldviews to impact decision making (Gersick, 1991). Culturally-imbued biases may degrade the quality of decision-outcomes within cc-TMTs because executives' judgments and decision-making processes can be prejudiced by their endemic cultural frames. Culturally-based mental models not only introduce new biases into the cc-TMT decision processes, but also amplify pre-existing cognitive biases that reside within each member. As conflicting cultural and cognitive biases grapple against one another across conference tables, team rationality erodes.

\section{Outsiders-Looking-in}

Cultures invariably look different from the outside-looking-in as opposed to the inside-looking-out. Absent intervention, executives' decision-making points-of-view are inevitably influenced to some extent by their cultural points-of-viewing. These dual conditions arise naturally and abide globally. Each offers threat or opportunity to executives who compete and collaborate in cross-cultural contexts.

Humans naturally "see" the world from the inside-out. Consequently, the ability to spot other-cultured executives" "irrationality" when their situational interpretations don't align with our "obviously rational" views comes easily. But human biases concurrently inhibit us from seeing our own irrational and often faulty views. One need only observe routine political or religious discourse to understand the degree to which intra-cultural or other-group biases are self-reinforcing. Or consider how, when their biases are challenged, most people instinctively erect one-sided, selectively rationalized "facts" - easily fabricating seemingly empirical and rational defense mechanisms as strongholds of self-confirmation. In this way, biases become self-rejuvenating and self-preserving. Biases tend to close eyes, ears and minds; inhibiting people's ability to openly receive or accept alternative wisdoms. The notion that, absent conscious intervention from themselves or others, even wise executives could ever see themselves from the outside-in is untenable.

Humans naturally defend and rationalize these inside-out views. We do so in part because having fixed mental models (i.e., biases) lowers uncertainty and simplifies a complex world. Certainties are highly valued by people operating in complex and risky contexts. To make progress in complicated business environments, merely human executives, like all of us, glom onto (and defend) their culturally-specific biases, stereotypes, and other assumptive heuristics. Thereafter, these biases are used to simplify, interpret and manage vast amounts of complicated environmental stimuli.

Learning theory suggests humans continue to do what previously provided good results, even when underlying conditions have changed (March, 1991). What worked before is usually repeated, a human propensity that prevails 
across cultures. Humans typically exploit what they already know and rationalize this knowledge is still valid even if the decision-making processes that result are no longer rational. Every investment prospectus diligently reports: "Past results do not guarantee future performance." But when considering their past successes, executives often ignore such admonitions. Cognitive inertia locks-in on pre-existing mental models. Open-minded, more rational thinking is limited if not prohibited.

To make rational decisions, executives must interpret relevant environments accurately. In process, however, their personal pallet of ideologies, experiences and perceptions - each shaped by cultural orientations - continuously colors interpretations. Yet if TMT members misinterpret environments, decision quality deteriorates. When cc-TMT members "rationally" misinterpret the styles and behaviors their other-cultured (and equally irrational) partners use during decision-making processes, team decision making will also prove more fractured and irrational. One cultural cohort's rationality may conspire such that it discerns irrationality's presence in cultural-others who, in their minds, are completely rational actors. During processes tantamount to an escalating stalemate, each cohort irrationally draws conclusions about its rationality while concurrently irrationally concluding that the other cohort is being irrational. If neither cultural cohort is aware of its biases, each may attempt to defend and rationalize the irrational.

Consider how the tenacious presence of Indian executives' cultural biases could introduce irrationality to and frustration with processes through which cc-TMT decision are deliberated. But the same "biased" Indian decision-making processes (from say, culturally-outsiders-looking-in British teammates) that shape decision outcomes appear predictably rational to Indians. Of course, from Indian perspectives, British decision-makers routinely manifest their own cultural biases. Culturally-diverse TMTs can easily be hijacked by irrational decision-making processes because cultural biases are ubiquitous and impact cognitive biases.

H.L. Mencken wrote: "For every complex problem, there is an answer that is clear, simple and wrong." Any argument that cultural-cum-cognitive bias is primarily responsible for irrationality in the decision making of otherwise rational cc-TMTs is likely too ambitious. However the argument just formulated merits as much or more consideration as any alternative explanation. Cultural and cognitive biases, as partial causal factors, logically synopsize why rational executives often act otherwise in cc-TMT decision-making contexts.

\subsection{Predictable Irrationality}

Behavioral economics theory (BET) is an emergent managerial force, in part because it explains why rational beings frequently make irrational choices. Financial managers, policy makers and marketers now routinely employ behavioral economic insights about the limits of human rationality to manage their professional efforts more effectively. Yet strategic-level decision makers interacting in cc-TMTs rarely account for the rationality-blocking biases that BET addresses (Lovallo \& Sibony, 2010).

One likely explanation is apparent. When financiers, marketers or public policy makers employ BET principals they need only recognize biases residing in others to predict causes for behavior. As always, inside-out views are easy. But before BET principles can be leveraged in cc-TMT interactions, executives first must recognize and account for their own biases. But critical outside-in-examination is challenging for most.

Inside TMTs, quality of process purportedly contributes more to quality of decision outcomes than does quality of analyses - by a factor of six (Lovallo \& Sibony 2010). This does not infer quality analysis does not matter. To the contrary, it reveals that robust decision-making processes are rarely accompanied by deficient analyses. Unbiased decision-making processes should flush out weaker analyses. The inverse is not true. Brilliant analyses can be rendered impotent if decision-making processes fail to yield rational scrutiny of viable options through unbiased hearings. Were cultural and cognitive biased better understood, executives could identify their biases before making flawed interpretations. The willingness to identify one's biases is a first step toward avoiding the irrationalities they can engender. If executives understood how other-cultured TMT members also engaged with their specific biases in tow, the whole team should benefit from the less biased decision-making processes that follow.

\subsection{Predictable Cultural and Cognitive Biases, Too}

Cultural biases - clannish views, habits, schema and heuristics engendered and reinforced by endemic cultures impose substantial impediments to rational decision making in cc-TMTs. Ties of history, shared experiences, and tribal-like customs and habits - biases each - explain not only who is more likely to do business with whom, but also who is more likely to partner with whom. Birds of a cultural feather, it seems, flock to do business together.

Cultural biases exist whenever decision-makers interpret experiences, events and/or information based on standards endemic only to native cultures. Shared cultural ties matter in business because they lower transaction costs by fostering higher levels of trust and reduced fears of opportunism. Similarly, in cc-TMT decision making, shared 
cultural biases supercharge positive communication due to their impact on trust and understanding. Differing cultural biases may have the opposite effect. Reading contracts are trusting they are ironclad is useful. But cc-TMT players also need to read and trust other-cultured participants who agree to and must execute those contracts.

Cognitive biases are unconscious, systematic tendencies that cause humans to stray from rational thinking. Because all humans share them, cognitive biases are easier to predict than cultural biases. Cognitive biases exist when notable deviation from decision-making processes and outcomes that others would normatively expect becomes evident. They function like mental traps pervasively contributing to less rational calculations and decisions, even among rational decision makers. When present, cognitive bias may affect decision outcomes and influence the degree of perceived rationality present in TMT's decision-making processes. In cc-TMTs, primary cognitive biases are triggered by, blended with, and compounded through interactions with cultural biases. As disparate cultures are mixed, the implications of now compounded biases become even more complex.

Five useful categories of cognitive biases are (Lovallo \& Sibony, 2010): (1) biases in pattern recognition; (2) stability biases; (3) social biases; (4) biases toward action; and (5) biases toward personal interests. "Pattern recognition bias" prompt decision makers to recognize or suspect certain patterns exist in data or environments, or to miss or ignore patterns that are present. "Stability biases" lead cc-TMT players towards more or less decision inertia as uncertainty rises. "Social biases" facilitate differing member perceptions of the value of harmony when contentious discussions occur. "Biases toward action" within cc-TMTs might drive one cultural cohort to initiate action based on more or less due diligence than cultural others prefer. "Personal interest biases" result when cohorts engage in silo-like thinking inside TMTs, where each side defends its values at the expense of holistic reasoning.

Table 1. Common cognitive biases executive decisions

\begin{tabular}{ll}
\hline Category & Manifestations \\
\hline Bias Toward Action & Excessive optimism \\
& Overestimating outcome probabilities \\
& Overconfidence/hubris \\
& Minimal concern for uncontrollables \\
Bias Toward Personal Interests & Skewness toward personal gains \\
& Emotional attachments \\
Pattern Recognition Bias & Skewed interpretation of key decision factors or the \\
& weighting of them \\
& Selective perception - only open to what confirms \\
existing beliefs & Taking lessons from inaccurate or incomplete analogies \\
Expecting past patterns to repeat in future conditions \\
Sticking to what is known in the face of change \\
Sunk-cost fallacy \\
If it isn't broken, don't fix it. \\
Fearing losses more than desiring gains (Prospect theory) \\
Avoiding prolonged conflict \\
Groupthink \\
Following the leader, because they are the leader
\end{tabular}

Adapted from Lovallo and Sibony, (2010)

Extolling a foundational BET premise, Dan Ariely suggests, "Not only are we [humans] irrational, but we are also 'predictably-irrational' in the same way, again and again" (2006; p. 20). If humans are not just irrational but predictably so, executives engaging in cc-TMTs need not view cultural-induced decision-making biases, their own or others', as insoluble problems. Instead, executives need only understand and prepare for predictable cultural biases that trigger predictable cognitive biases.

\subsection{Context: Three Dominant Cultures}

Fossil fuel issues notwithstanding, three cultural networks (i.e. spheres) dominate the contemporary global economy. Each sphere emerges from infrastructures grounded in culturally-specific worldviews and values. Each worldview 
has been shaped by specific points of pride and prejudice, unique histories of legendary victories and humiliating defeats, and culturally-specific shadings of past evils, venality and disorder.

The world's oldest continuous cultural network, the Sino-sphere, is currently its most vibrant. The global influence of Chinese culture is reinforced though links with the some 70 million ethnic Chinese living outside China. Many descend from individuals who moved abroad during China's imperialist expansion from the $12^{\text {th }}$ to $15^{\text {th }}$ centuries. Their predecessors settled and imbued Chinese cultural values into regions that since morphed into Asian tigers such as Vietnam, Malaysia, Myanmar, Singapore and Indonesia. More recently, Chinese businesspeople, investors and students have migrated by the millions to the U.S., Taiwan, Hong Kong, Canada and various African nations.

Another of the world's rising cultural networks, the Indo-sphere, emerged more through organic means than centralized processes because of its democratic underpinnings. Ethnic Indians comprise less than 1\% of the U.S. population, but account for $13 \%$ of graduate students at America's elite universities. In Great Britain ethnic Indians earn at least $10 \%$ more than the average income. In the U.S., Indian households boast the highest median income of any ethnic segment. The top five destinations for Indians' international investment each boast large émigré Indian populations: the U.S., Great Britain, United Arab Emirates, Singapore and Mauritius. Tata, an Indian conglomerate led by a British-educated executive, employs more manufacturing workers in Britain than any other firm.

Clearly suffering from years of iterating crises in confidence and more tangible factors, yesteryear's bullyboy still packs a punch. The Anglo-sphere mightily influences global business and diplomacy, although no longer at dominant levels of the past. Trading and cultural links between Great Britain and its former colonies (the U.S., Canada, India, Hong Kong, Singapore, Ghana, to note a few) indicate tight tribal connections. Trade flow rates between Britain and its former colonies are $13 \%$ higher than chance would prescribe, capital flows $24 \%$ higher, and, remarkably, flows of people and information 93\% higher. English remains the world's lingua franca - wielded reflexively and systematically to make communication possible between people who do not share a common language. This alone underscores the Anglo-sphere's global business impact (Wooldridge, 2012).

While the three spheres increasingly overlap as technology flattens business and social worlds, each sphere's unique cultural history injects radically differing biases into cross-cultural interactions. Resulting misperceptions and misunderstandings can prove dramatic when executives from any two of these three cultures interact. Yet for two reasons we focus on Sino- and Anglo-spheres as pressing cultural biases are explicated and prescriptive managing bias rules are prescribed below. First, India once was a British colony. India unquestionably benefitted economically and has been changed permanently from the imposition and ultimate integration of certain British rules, language, customs and values. That idiosyncratic Indo values could be teased out as wholly independent from the Anglo-sphere's business values is doubtful. The second reason is that the Sino- and Anglo-cultural spheres are currently more globally determinant and economically tied to each other than either is with the Indo-sphere.

\section{3. cc-TMTs: Where Cognitive and Cultural Biases Collide}

In one way or another, all humanity is biased. Experts also suggest everyone at times is irrational; indeed, predictably so. Consequently, not only do differences in their dominant cultural beliefs, values and historical/economic backgrounds prompt Sino- and Anglo-executives to lean more readily into certain culturally-encoded biases, these differences also prompt culturally divergent executives to lean more readily into certain predictably-irrational cognitive biases as they work toward decisions in cc-TMT settings.

No reason exists to suggest one culture's biases are more right or wrong than others. But various reasons exist to suspect that disparate cultural biases may play out in ways that compound cognitive biases. These second-order cognitive biases, in turn, impact cc-TMT decision-making processes. This progression is managerially significant. The presence of culturally-driven cognitive biases in cc-TMT settings may contribute to decision-making processes and outcomes that other-cultured team members perceive as illogical, inappropriate and irrational.

The value of diverse executive teams has long been recognized. Mary Parker Follett, born in 1868, wrote: "Contentious problems are best solved not by imposing a single point of view, but by striving [for solutions] that integrate the diverse perspectives of all relevant constituents." Yet if cultural diversity-induced biases are not managed, swirls of misaligned cultural and cognitive frames that coincide and collide inside cc-TMTs may prove so irrationally influential that the potential value of diverse executive perspectives is undermined. This prospect alone should motivate cc-TMT participants to become better informed about the sources, nature and consequences of, and possible treatments for culturally-influenced cognitive biases.

Development of quality cc-TMT decision-making process, as prerequisites for rational decision making, generally inspires debate. Dialectical and devil's advocacy approaches to evaluating alternative interpretations and 
recommendations are often necessary to improve team decision-making. But each approach inherently engenders conflict. If comparatively less biased and more open decision-making processes are not established as normative means through which disputes are addressed, less rational decision outcomes become more likely. When cultural and cognitive biases remain unacknowledged and untreated, behaviors are more likely to be interpreted as parasitic, i.e. one cohort engaging in what the other deems more take-than-give. Irrational or ambiguous behaviors may also be interpreted as signs of hidden agendas. Trust is injured as the willingness to invest resources mutually or pursue naturally risky innovation diminishes (Simons \& Peterson, 2000).

\section{Same World; Different Worldviews}

Sino and Anglo executives connect with vastly differing worldviews in place. Sino decision-makers are culturally biased by their legacy exposure to ancient Chinese philosophies (Confucianism), prediction practices (I-Ching) and ideologies (Taoism). Consequently, they tend to seek and value deliberative decision making, harmony and a collectivistic perspective. Sino executives are predisposed toward patient decision making. Longer-term outcomes thus enjoy high priority. Sino executives are also receptive to using strategic guile or purposefully coloring information to pursue sub rosa goals (Strutton, Tran and Taylor, 2012). Similar culturally-driven cognitive biases have influenced Sino decision making for millennia (Du, Ai, \& Brungha, 2011; Hsu \& Chiu, 2008).

Decision makers from Anglo-spheres arrive having been deeply influenced by latent Judeo-Christian theologies and cultural upheavals like the Reformation and the Enlightenment. Stemming from the high value assigned to personal liberties, Anglo-spheres emphasize individualism and self-determination. Cultural individualism is manifested in a cognitive focus on the importance of personal needs, justifiable rights and individual freedoms (Chow, et. al, 1999). Western economic theory and business practice is grounded in cultural presumptions about the risks of opportunistic and self-interested behaviors of others. Ensuing "protective-of-me-and-mine" motives impede information sharing if disclosure is not deemed in one's best interests. Anglo decision making is usually personally calculative. The sphere also allows for freedom of assertiveness and independent expression, particularly as compared to Sino tendencies to value cohesion-centric/collectivistic behaviors.

Sino and Anglo worldviews differ in various practically significant ways. Yet inside cc-TMTs their diverging worldviews not only must interact with each other, but also with cognitive biases. The interactions can hinder cc-TMT rationality, as discussed below.

Table 2. Cultural-biases and behaviors toward key decision process elements

\begin{tabular}{|c|c|c|c|}
\hline Element of TMT & Key & SINO & ANGLO \\
\hline Decision Process & Issues/Cognitive Biases & Biases & Biases \\
\hline \multirow{3}{*}{$\begin{array}{l}\text { Interpretation of the } \\
\text { Environment }\end{array}$} & Temporal horizon & Long-wave & Short Wave \\
\hline & Value of approach & Quality of judgment & Speed of judgment \\
\hline & Heuristics & Holistic, broad view & Narrow, analysis rules applied \\
\hline \multirow{3}{*}{$\begin{array}{l}\text { Response to Uncertainty } \\
\text { and Change }\end{array}$} & Complexity & Embrace it & Attack it \\
\hline & Uncertainty & Deny ability to control & Strive for control \\
\hline & Attitutde toward change & Opportunity in change & Risk and cost in change \\
\hline \multirow{5}{*}{$\begin{array}{l}\text { Debate, discussion, and } \\
\text { decision making }\end{array}$} & Inform gathering & Synergistic collective & Zero-sum individualistic \\
\hline & Decision speed & Timing must be right & Timing is asap \\
\hline & & Comtemplative & Action, action, action \\
\hline & Discussion and debate style & Respect/non-confrontational & Competition \\
\hline & & Harmony seeking & Victory seeking \\
\hline
\end{tabular}

4.1 Environmental Interpretations: Biases in Action, Patterns, and Recognition

To succeed, cc-TMT executives must accurately interpret relevant external environments and pursue all reasonable (i.e., affordable) clarity regarding the current status of customers, suppliers, partners, competitors, regulations, etc. Executives also must assess and predict future trends associated with various factors that influence strategic choice. Pattern recognition, obviously, is crucial to success. But success becomes less likely if the interpretative process is confounded by misaligned and unaccounted for cultural biases.

Sino-centric executives appear more likely to adopt holistic views of the environment. Decision factors are likely interpreted within collectivist frameworks rather than ones grounded in self-interests. Issues are viewed and treated as individual component elements within larger systems of interdependent actions and reactions. Longer-term, 
broader perspectives follow. Given these expansive views, Sino-centric executives likely simultaneously consider/evaluate multiple interpretations of events and alternative explanations for cultural others' motives (Haley $\&$ Haley, 2006). In Sino-centric circles, quality decision-making is best achieved by patiently contemplating multiple scenarios and possible outcomes.

Anglo-centric interpretative approaches are usually shorter-term and action-oriented. Rapid-fire decision making follows naturally. Rather than enlisting holistic, system-wide views, problems are typically isolated to resolve them as quickly and efficiently as possible. Action biases generally prevail. Anglo-centric executives value the speed with which they can make critical decisions and initiate actions in desired directions; directions often leaning toward fulfillment of individual rather than collective interests. Consequently, Anglo decision makers often employ decision rules, frameworks or heuristics based on past or analogous patterns to interpret and navigate environmental uncertainty (Haley \& Haley, 2006). Anglos typically consider fewer alternatives as competing options are quickly eliminated through an expeditious process of evaluation.

Environmental interpretations also involve evaluation of others' communication and behavioral patterns. This matters because in cc-TMTs where none of their intrinsic cultural biases were brought to light, Sino and Anglo executives each would tend to assume customers, competitors, suppliers and cc-TMT partners are driven by the same motives as them. These mistaken attributions of familiar/similarly-biased views further diminish the prospect that rational environmental interpretations will be achieved. As discrepant perspectives coincide and collide inside cc-TMTs, reconciliation of conflict and finding agreement on goals and environmental interpretations can prove difficult.

For example, alternative temporal perspectives, each emanating from differing biases toward action, may appear irresponsibly short-sighted to Sino members but unnecessarily time-consuming to Anglos. Whereas Sino-sphere executives are biased toward evaluating multiple alternatives within interdependent systems, Anglo executives are biased to quickly accept satisficing decisions and then initiate action. To Anglos, Sino partners may appear non-committal, overly cautious, and fearful of making a decision. To Sinos, Anglo cohorts may appear reckless, inflexible, and condescending. Yet each cohort views its interpretations as rational. Responsibility for irrationality lays with the cultural other. Ultimately, attempts at understanding the irrational behaviors of seemingly intelligent cultural others may prompt trust-reducing beliefs that hidden agendas are in play (Simons \& Peterson, 2000).

\subsection{Stability Interpretations: Biases toward Uncertainty, Complexity, and Change}

Successful decision-making processes in cc-TMTs require coordinated management of uncertainty, risk, complexity and change. Such factors loom would large in the intrinsically dynamic decision-making processes executed inside cc-TMTs. Again, deep structures associated with their divergent cultural orientations may propel Sino and Anglo executives to approach the interpretation and management of these factors with differing biases in place. Stability biases, unchecked, would leave executives more or less likely to depart from the status quo even when rationality or logic suggests they should shift strategically in one or another direction.

Sino executives are culturally conditioned to embrace uncertainty and risk as natural non-threatening conditions. They generally deny that control over the fates is even achievable. Complexity, in Sino-spheres, is absorbed by engaging others to reduce the influence of personal interest bias and selective pattern perceptions (Boisot \& Child, 1999). Sino executives enter cc-TMT settings linked by collectivist biases. They are habituated to value networked social approaches, advice-seeking, and counsel from respected others (Zhang \& Chua, 2009). Biases toward contemplation over quick action lead to additional information gathering as additional options are considered. Uncertainty and complexity are neither feared nor avoided. Instead, each is embraced as opportunity.

Anglo executives seek to control complexity and manage risk (Boisot \& Child, 1999). Individualistic and self-deterministic cultural shadings generate views that even as the world changes, people can still control their destinies through purposeful action. This bias can lead to overconfidence in the face of uncertainty and risk. Action-bias is often accompanied by pattern recognition-bias; i.e., believing that past experiences inevitably relate to new situations. In Anglo-spheres, mastery of ones' environment is generally viewed as crucial to success. Uncertainty and complexity are seen as threats to stability and control. Cultural predispositions to confront, control or eliminate uncertainty and complexity tend to dominate (Haley \& Haley, 2006). To the uninitiated, biases toward the value of stability and control may appear primed to lead Anglo executives to avoid risky activities. In fact, the opposite is more likely. Biases can prompt more risk-taking as hubristic false senses of security emerge and overly optimistic interpretations are acted on. 
As the two "tribes" work through cc-TMT-level issues and challenges that inevitably involve management of change, uncertainty and complexity, Anglo cohorts may view Sino partners as tepid or fearful. Essentially, the perception is that they cannot make up their minds. Sino cohorts may be less likely to actively assert their perspectives in such a context. Unfortunately, such non-confrontational behaviors may be interpreted by Anglos as indecisiveness, hedging, lack of commitment to a plan, or worse still, incompetence. In turn, Sino cohorts may view Anglo behaviors as brash, arrogant or unenlightened - or merely naïve and undeserving of respect. Worse yet, should Sino cohorts sense their cautious consideration (i.e., to them, due diligence) or traditional approaches are castigated by Anglos, they may perceive this as a lack of respect for the legitimacy of thousand-year-old methods and traditions (Strutton, Tran and Taylor, 2012). As inaccurate judgments mount, prospects for cc-TMT cohesiveness, cooperation and rationality - in present or future tense - slip away.

\subsection{Informational Interpretations: Biases in Social Processing, Discussing, and Debating Styles}

cc-TMT participants must interpret and process information accurately to facilitate effective decision making. This is a necessary precursor to any cc-TMTs' subsequent ability to successfully discuss/debate alternative interpretations and various courses of action. Differing informational interpretative styles, which at their cores entail differing social biases, influence how Sino and Anglo cohorts approach and respond to one another during discussions.

As noted, Sino participants are culturally predisposed to collectivistic interpretations. This implies they assign higher values to more interdependent social views. Even cultural others should be honored and respected for their individual wisdom and loyalties. Verbal challenges are avoided. Sino executives tend to involve others in their decision-making and to seek advice rather than taking and defending independent positions. Biases toward the non-confrontational prevail because harmony is so highly valued (Du et al., 2011). Their cultural values bias Sino participants to consciously pursue harmonious consensus, but only after considerable reflection and integration of multiple, holistic views has transpired. Debate and discussion should remain cool, calm, balanced - literally collected (Zhang \& Chua, 2009). Once a decision is made, Sino executives' holistic biases often motivate new concerns that all implementation actions are precisely ordered. Timing is viewed as a critical component of a rational action plan.

Anglo culture, as described, stresses individualism and competition. While a Sino-like social bias toward avoiding confrontation may increase risks of groupthink, thorough debates on key issues can become contentious zero-sum contests resulting in winners and losers. Anglos are generally predisposed to more forcefully assert individual interpretations/conclusions and defend positions when opposed. Final decisions often emerge from Anglo-led processes only after one alternative has survived these rigorous challenges. Healthy task conflict can easily degrade into harmful relational conflict if trust fades (Simons \& Peterson, 2000). Grounded in their biases toward stability, Anglos who sense they hold "losing hands" during debates may become defensive and introduce irrational barriers to change. With respect to timing, once decisions have been made, Anglos are biased to believe the faster implementation begins the better.

\subsection{Net Cognitive Biases}

Differences in deeply-held cultural views about how TMT decision-making should proceed can prompt Sino- or Anglo-executives to assume the other cohort's perspectives are irrational. This appears particularly likely when neither cohort is sufficiently aware of the other-cultured cohort's cognitive biases. For instance, if they are the weaker cohort in cc-TMTs, Anglo participants might become frustrated and disillusioned - bending them toward the irrational - by the perceived tentativeness and complexity-absorbing tendencies that drive Sino-led decision-making. At one extreme, Anglos may respond with independent, uncoordinated decisions. Cultural biases toward action and speed might prompt Anglos toward act-now, ask-for- forgiveness-later approaches. In Sino views, Americans, at least, would be acting out culturally-stereotyped "cowboy" styles. At another extreme, Anglos may simply withdraw from the discussion. Anglo cultural/cognitive biases toward stability and self-interests then may be viewed by Sino counterparts as stubbornness and lack of cooperation.

By contrast, when Sino cohorts occupy weaker positions, they may perceive Anglo-led decision-making processes as too aggressive and self-assured (i.e. egotistical and arrogant). Anglo initiated action-biased decision making might strike Sino counterparts as overly reliant on no-longer-relevant experiences. As Anglo-led processes unfold during shorter evaluation cycles, Sino executives may feel harried into accepting decisions before acceptable levels of consensus exist. This can prove troubling, for the prospect of being accountable for decisions made before consensus is achieved is emotionally burdensome for Sino partners. Less-than-ideal levels of commitment to and implementation of decisions may result. Confounding matters further, culturally-imbued Anglo biases toward more aggressive debating styles may prompt tacit withdrawal by Sino members who value group harmony and 
non-confrontational styles. Withdrawal, in turn, only reinforces Anglo biases, given their cultural drive to compete vigorously even during battles of styles.

Human cultural and cognitive biases, and the decision-making processes each affects, are hugely complex and dynamic. Certain broadly generalized cultural biases were just introduced. But even if these broadly-framed factors are low in magnitude or manifested only occasionally, each instance can severely hinder rationality. In cc-TMTs the interplay of each cohort's cultural/cognitive biases with the "other's" biases likely begets additional, iteratively more-biased responses. This cascade of biases may lead each cohort to pursue divergent decision-making processes tomorrow that are as depressingly flawed as the ones that failed yesterday.

\section{Rules for Reducing Bias in cc-TMT Decision-Making}

The goal of reducing the impact of cultural and cognitive biases in cc-TMT decision making is elusive and challenging. But this goal is also attainable and enticing - driven by a logical desire to deliver more rational decisions. If the quantity and influence of heretofore unacknowledged biases decrease, the frequency of rational decision process outcomes should increase. cc-TMT executives can consciously choose to succeed together by deciding more rationally, or, despite myriad global challenges pressing upon them (ensuring each surely needs the other) they can irrationally decide to fail as biased individuals.

The first step to solving any vexing problem entails acknowledging its existence and defining its nature, scope and causes. This article focuses on the iterative interaction of decision-making processes and cultural/cognitive biases, and wraps itself around one complicating problem. However, two simplifying insights are evident. First, there could never be enough rules to address all possible biased decision-making contingencies inside cc-TMTs. And second, to reduce biased decision-making processes in cc-TMTs, executives should

\section{- Never Get Hung Up Trying to Learn a Lot of Rules}

Rather than becoming rule-driven, cc-TMT executives should instead consciously acknowledge that each cohort is characterized by its own uniquely rational (to its culture) biases. Executives should also acknowledge that their own culturally-rational biases often become irrational in the blended cc-TMT's culture that each must share. Executives should then commit to learning what these differences in cultural biases are, why these differences exist and, how these differing biases negatively impact decision-making processes.

Each question has been answered above. The insights embedded in the answers may prove sufficient to nudge cc-TMT executives to subtly but deliberately adjust their presentations and expectations of self. As important, the insights may also prod executives to subtly but deliberately adjust their interpretations and expectations of cultural others. Even nuanced adjustments in expectations, interpretations, and interactions should reduce the onset, arrival and irrational influence of cultural and cognitive biases.

But bias-reducing rules were promised, and rules follow. Each broadly-framed rule is designed to reduce biased decision-making processes in cc-TMTs, especially if applied in the order presented.

Table 3. Rules for reducing the influence of bias in cc-TMT decision making

\begin{tabular}{ll}
\hline Rule & Key Elements \\
\hline 1. Seeking Behavioral Integration & Revisit and reinforce shared purpose \\
& Enhance social integration \\
& Increase acculturation \\
2. Appoint a Process leader & They are the guardians against biases \\
& The team should identify and appoint their leader \\
& A Collectivist with tenure is likely best suited to the rule \\
& Biases can't be eliminated, but they must be accounted for \\
3. Use a Process that Accounts for Biases & Leverage biases when appropriate \\
& Structure discussion rules and process to manage conflict \\
& Individual purposeful reflection \\
4. Learn Continuously & Postmortems to learn from success and failure \\
& Reflection before and during process to maintain awareness of \\
& influential biases \\
& The path toward perfection is imperfection \\
& Manage expectations of comprehensiveness \\
5. Embrace the Paradox of Perfection & Avoid hubris and habit
\end{tabular}




\section{Rule 1: Honor the Core Value of All TMTs: Behavioral Integration}

cc-TMT membership should begin by remembering why teams are developed and used in the first place. Teams offer forums through which people possessing diverse insights and competencies can synergistically accomplish more collectively than they could individually. Such outcomes become more plausible when members agree on common purposes and processes. Behavioral integration measures the level of a TMT's collaborative behavior, quality and quantity of its informational exchange, and degree to which members employ joint decision making (Hambrick, 1994). The construct captures the effectiveness of team member interaction. Successful behavioral integration within cc-TMTs follows from a shared vision, social integration, and mutual acculturation.

Diversity can facilitate superior decision-making inside cc-TMTs. (This is true for all teams.) Or, diversity can impose cultural/cognitive biases that weaken or destroy team solidity and greatly constrain decision-making effectiveness. cc-TMTs always face conscious choices to exploit diversity or to permit diversity to exploit them; or to live out shared good fortune or succumb to voluntarily shared misfortune. cc-TMT membership should deliberately focus on establishing convergent aims rather than diverse cultures, personalities and biases.

Given that multiple agendas are always in play, an explicit focus on a shared purpose is even more vital to sustain behavioral integration inside TMTs. To keep biases at bay or deny them traction, cc-TMTs should continually revisit and reinforce the team's shared vision. Drawing attention to common problems, threats or enemies can heighten collective focus. cc-TMT membership should never assume the positive force of "shared purpose" remains perpetually present. As each meeting begins, agenda items should underscore the identity and value of this shared purpose. Unity and team identity are crucial. Each should be nurtured.

Paradoxically, the individual human value of team members should also be made explicit. To exploit diversity in ways that reduce bias, each individual team personality should be recognized as valuable. Human relations are often better developed though social interactions occurring outside of immediate business settings. Such efforts should be initiated by the host cohort in less formal (albeit still structured) pre-meeting venues. Enhanced interpersonal relationships can lessen the prospect that biases will prompt knee-jerk irrational interpretations during debates. No need exists for everyone to be close friends. However as relational foundations based on personal respect, mutual understanding and other-awareness grow, less biased communication and decision-making processes should tag along. Informal social time spent together should help members view each other as unique, rather than stereotyped, individuals. Yes, my Sino partner is different, but we share similar desires and concerns (nice vacations, concerns about kids). Yes, my Anglo team member is smart, capable, worthy of respect; she brings unique value to the table and feels the pressure of her position - just like me. Social integration has always been a hallmark of successful business practice. Yet in ways large and small, the contemporary world's electronic pace cuts the face time necessary to achieve successful social integration. As screen time rises, direct human-to-human interaction falls proportionally. Individual personal relations still contribute significantly to inhibiting the influence of cultural biases. Face time must be created to know one another as individuals.

Mutual acculturation should enhance cc-TMT rationality. Mutual acculturation implies each cohort has recognized and internalized the diverse ways in which the cultural other approaches uncertainty, decision-making and the world. Insights can emerge through working and socializing with other-cultured teammates in receptively inquisitive ways. Insight could also grow through cultural experience. Each cohort should make room for "in-culture time". cc-TMT meetings usually require that one cohort travel to the "other" culture, presumably reciprocally over time. Parties should purposefully exploit visitation rights. Experiencing other cultures endows people with a valuable open-mindedness (anathematic to bias) (Maddux \& Galinsky, 2009), making it easier to experience issues and problems from multiple (inside-out; outside-in) points of view.

Consider simple cultural biases toward leaving food on one's plate. Chinese view this as a complement; the host has provided more than enough. Anglos may view the same act as distressing or insulting; the food was not appropriate or good enough to eat. Their learned open-mindedness means that seasoned travelers become more alive to ambiguity, willing to realize how different (but equally valid) ways of interpreting the world logically can exist - and co-exist. Examining alternative possibilities becomes more natural as the circumference of their cognitive experience expands in lockstep with the diminution of their bias. As executives learn different cultures, they will know more about what they do not know. The world truly has globalized. But at best its inhabitants continue to merely slouch toward similarity, even in situations where similarity in is desirable. Much needs to be learned about avoiding and managing cultural biases. Growing the head of a smart stranger is a logical place to begin. 


\section{Rule 2: Identify and Appoint a Process Leader}

All cc-TMT members share responsibility and accountability for understanding their own biases. Similarly, they are responsible for understanding cultural others' biases as they arise. Yet effective management of decision-making processes also requires that one team leader be appointed for decision-making process quality control. Process leaders act as guardians against cultural and cognitive biases that arise during decision-making deliberations.

The person holding the most legitimate or referential influence/control over final decisions is likely not the individual who should administer the process through which decisions are developed. Non-prejudicially leading cc-TMTs through unbiased decision-making processes is not part of most leaders' tool kits. This task requires particular leadership competencies.

The full team should be tasked to identify its process leader. This reinforces the core value of Rule 1; developing behaviorally integrated teams. Process leaders should have a collectivist orientation and extensive tenure in the relevant field (Chow et al., 1999; Simsek, Veiga, Lubatkin, \& Dino, 2005). More experienced process leaders should be better prepared to facilitate and guide unbiased discussions that extract, challenge, expand or clarify only that information most relevant to the decision context. Process leaders should willingly subordinate self to team and ensure members share, integrate, and process information in a collectively unbiased fashion. An integrative rather than domineering communication style is required. Their natural cultural biases suggest Sino executives generally arrive better prepared than Anglos to perform as process leaders.

\section{Rule 3: Implement Rational Processes that Account for and Exploit Biases}

Structured decision-making processes should be collaboratively developed and embedded in formal operational procedures that generate synergistic value from the integrated exploitation of certain cultural and cognitive biases that arise within cc-TMTs. But in delicate balancing acts, these same procedures should simultaneously countermand mechanisms that would promote bias-induced irrationality inside the team. This activity also heightens awareness of biases and of the value of a shared vision and behavioral integration within the cc-TMT. Rule 1 is further reinforced. Simply acknowledging that cultural biases exist within cc-TMTs need not trigger efforts to eliminate them. Instead the awareness should motivate teams to marry - integrate - "best practice" Sino- or Anglo-cultural biases in ways that enable the entire team to consciously pursue new synergistic values. Still, simply understanding that differing cultural biases exist may not lead executives to recognize how the intersection of these biases inside the cc-TMT might allow them to enjoy the best of both worlds. The potential opportunity to exploit integrated biases should be persistently emphasized by process leaders. Executing some decisions by fostering the dominance and exploitation of one culture's bias at the other's expense at times is rational. For instance, in crisis situations with impending deadlines, Anglo biases toward rapid-fire evaluation and action may prove optimal. Sino-executives - who are biased toward contemplation - should sublimate their cultural predilections for the team's sake.

As alternative approaches to making critical decisions are developed and evaluated, discussion often degrades into debate. In relatively stable environments, comprehensiveness (i.e., thorough diagnosis of problems and generation/evaluation of alternative solutions) positively impacts firm performance (Fredrickson, 1984). But debate and competition among differing assumptions, conjectures and positions is required to achieve comprehensiveness. Meanwhile, consensus and harmony are useful precursors to successful behavioral integration. Another balancing act comes into play, because positive conflict associated with debates emanating from devil's advocacy or dialectical inquiries similarly facilitates superior decision-making processes (Amason, 1996).

One risk, especially when opposing biases are involved, is that uncontrolled conflict in otherwise constructive debates regresses into destructive relational conflict (Simons \& Peterson, 2000). To ensure productive deliberations, processes (and process leaders) must manage personal interactions. To depersonalize debating processes, generate open-mindedness and promote shared purpose, the format of decision-making processes should be preemptively and explicitly established by the team. Effective and positive debate would rarely unfold organically simply because executives are "professionals". Prospects that debates can be successfully orchestrated through fair and balanced processes would be bolstered by openly acknowledging biases before they negatively influence deliberations. Nobel Economist Daniel Kahneman suggested "The odds of defeating biases in group settings rise when discussion of them is widespread." More so than rational people, rational processes are likely to elevate decision making safely above dangerous whirlpools of cultural and cognitive biases.

\section{Rule 4: Learn}

When cc-TMT executives gather to make decisions, opportunities arise to learn more about their biases, those of teammates, and how each cohort's biases interact to promote or hinder rationality. Like emotions, biases are natural 
and intimately human attributes. Any assumption that any cc-TMT could completely eliminate biases and their influence is irrational. But notions that team executives could identify and learn from prior situations in which biases were successfully/unsuccessfully managed are completely rational. Process leaders should conduct postmortems of what went right or wrong with prior decision processes. Those biases more likely to impact critical decisions could be outed, ideally through open team discussion. Scrupulous observation of current decision-making processes would serve similar purposes as tacit knowledge about how to develop more rational decision-making process grows.

Still, no panacea will likely ever exist. Indeed, new and unintended procedural inefficiencies may arise as explicit effort is expended to manage decision-making biases. But internalized virtuous cycles of improvement in cognitive habits and correspondingly less biased decision-making behaviors may be fostered as intentional learning occurs. Given the proper team climate, such learning could occur absent conscious thought (similar to how cultural biases are learned). Such learning could emerge from purposeful reflection conducted before, during and after decision-making processes. Purposeful reflection, i.e., reflexivity, significantly improves team performance (Gurtner, et. al., 2007). Executives could reflect on prior team interactions before beginning new process sessions, recalling how decision-making processes "allowed" certain biases to emerge or prevented biases from irrationally influencing decision outcomes. During decision-making processes, targeted questions and open discussions could be facilitated by process leaders during breaks to promote reflection - and learning.

\section{Rule 5: Embrace the Paradox of Perfection}

One inherent paradox exists inside each cc-TMT - or domestic TMT, for that matter. Such paradoxes do not exist to be solved or reduced. They instead exist to be embraced as unavoidable realities - similar to how Sino-sphere players more readily embrace uncertainty. Lucilio Vinani (1585-1619) introduced the paradox of perfection, which suggests perfection requires imperfection. That is, were perfection achieved, no opportunity would exist to change, grow or adapt; problematic because change, growth and adaptation are necessary to sustain success. Therefore, perfection must embrace imperfection.

The paradox of perfection relates to cc-TMT decision-making processes because cognitive and cultural biases change over time. As certain biases recede, others inevitably advance. Team members come and go. Business conditions change. Strategies and priorities evolve. The pursuit of perfectly rational decision making also must continuously evolve. Healthy cc-TMTs embrace change and environmental irregularities as opportunities for progress.

cc-TMT executives should likewise embrace the reality that rationality is limited, accepting and acting based on the paradox that focusing exclusively on rationality is itself sometimes irrational. As noted, decision comprehensiveness is critical. Yet comprehensive decision-making is also paradoxical insofar as comprehensiveness is most valuable when environmental stability and certainty are high. When uncertainty and instability are high, conditions are typically too complex or dynamic to reward investments in the time required to conduct thorough analysis. In some cc-TMT contexts, the rational first choice is to determine the appropriate level of comprehensiveness to pursue and manage time accordingly.

Other paradoxes exist in cc-TMT decision-making contexts. For example, prior success and achievement paradoxically offer little value in dynamic cross-cultural environments. Indeed, as cc-TMTs create additional rational decision-making processs, cognitive and process rigidity are more likely to take hold inside the team. The hubris, complacency and overconfidence that often accompany team success can magnify the impact of irrational "slips". The presence of rationality should increase recognition of biases and their traps, rather than assumptions that biases have somehow been permanently conquered. The racer's paradox suggests people often move faster by moving slower. Taking time to avoid traps and missteps can prove less costly - time consuming - than recovering from mistakes of speed and confidence.

\section{Using the Rules: How and Why}

Each rule should partially alleviate irrational decision-making in cc-TMTs. But cc-TMTs - and their relevant business sectors, relevant environments and decision contexts - invariably differ. Certain teams are more vulnerable to the effects of cultural and cognitive biases than others. No one-size-fits-all method exists to ensure more rational decision outcomes. Thus, the magnitude of importance or degree of fit for any rule may wax or wane across decision-making contexts. Cultural and cognitive biases are interdependent. Similarly, these rules amplify and play off one another; they are interdependent. Degrees of cc-TMT rationality will expand only after the entire integrated system of rules has been implemented. No single rule or subset of rules introduced in part or independently would suffice to address interactive cultural and cognitive biases as they unfold inside cc-TMTs. 
Cultural biases hardly explain everything, but they explain enough in today's world that ignoring their effects is foolish. When biases infect a cc-TMT, executives overly embedded in their own cultural biases too often irrationally ignore or reject good ideas produced by cultural outsiders. During an era when many executive teams are taking real-time, high-stakes tests on how well they can manage diverse cultural biases, the need to learn how to negate and/or leverage the legacy power of these unavoidable biases is more pressing than ever. The rules provide useful insights regarding how the irrational decision-making processes often provoked by cultural and cognitive biases interacting inside cc-TMTs may be improved.

\section{References}

Amason, A.C. (1996). Distinguishing the effects of functional and dysfunctional conflict on strategic decision making: Resolving a paradox for top management teams. Academy of Management Journal, 39(1), 123-148. http://dx.doi.org/10.2307/256633

Ariely, D. (2010). Predictably irrational: The hidden forces that shape our decisions. New York: Harper Perennial.

Boisot, M., \& Child, J. (1999). Organizations as Adaptive Systems in Complex Environments: The Case of China. Organization Science, 10(3), 237-252. http://dx.doi.org/10.1287/orsc.10.3.237

Chow, C.W., Harrison, G.L., McKinnon, J.L., \& Wu, A. (1999). Cultural influences on informal information sharing in Chinese and Anglo-American organizations: An exploratory study. Accounting, Organizations and Society, 24, 561-582. http://dx.doi.org/10.1016/S0361-3682(99)00022-7

Du, R., Ai, S., \& Brugha, C.M. (2011). Integrating Taoist Yin-Yang thinking with Western nomology. Chinese Management Studies, 5(1), 55-67. http://dx.doi.org/10.1108/17506141111118453

Fredrickson, J.W. (1984). The comprehensiveness of strategic decision processes: Extension, observations, future directions. Academy of Management Journal, 27(3), 445-466. http://dx.doi.org/10.2307/256039

Gersick, C.J.G. (1991). Revolutionary change theories: A multilevel exploration of the punctuated equilibrium paradigm. Academy of Management Review, 16(1), 10-36.

Gurtner, A., Tschan, F., Semmer, N.K., \& Nagele, C. (2007). Getting groups to develop good strategies: Effects of reflexivity interventions on team process, team performance, and shared mental modes. Organizational Behavior and Human Decision Processes, 102, 127-142. http://dx.doi.org/10.1016/j.obhdp.2006.05.002

Haley, U.C.V., \& Haley, G.T. (2006). The logic of Chinese business strategy: East versus West: Part 2. The Journal of Business Strategy, 27(2), 43-53. http://dx.doi.org/10.1108/02756660610650037

Hambrick, D.C. (1994). Top management groups: A conceptual integration and reconsideration of the "team" label. Research in Organizational Behavior, 16, 171-213.

Lovallo, D., \& Sibony, O. (2010). The case for behavioral strategy. Retrieved November 5, 2011 from https://www.mckinseyquarterly.com/The_case_for_behavioral_strategy_2551

Maddux W.W., \& Galinsky, A.D. (2009). Cultural borders and mental barriers: The relationship between living abroad and creativity. Journal of Personality and Social Psychology, 96(5), 1047-1061. http://dx.doi.org/10.1037/a0014861

March, J.G. (1991). Exploration and exploitation in organizational learning. Organization Science, 2(1), 71-87. http://dx.doi.org/10.1287/orsc.2.1.71

Simons T.L., \& Peterson, R.S. (2000). Task conflict and relationship conflict in top management teams: The pivotal role of intragroup trust. Journal of Applied Psychology, 85(1), 102-111. http://dx.doi.org/10.1037/0021-9010.85.1.102

Simsek, Z., Veiga, J.F., Lubatkin, M.H., \& Dino, R.N. (2005). Modeling the multilevel determinants of top management team behavioral integration. Academy of Management Journal, 48(1), 69-84. http://dx.doi.org/10.5465/AMJ.2005.15993139

Wooldridge, A. (2012). The Power of Tribes. The Economist, 402 (8729), 68.

Zhang, Y., \& Chua, S.K.C. (2009). Influential leadership: A Harvard model vs. an I-Ching model. Chinese Management Studies, 3(3), 200-212. 